\title{
Pattern of smoking cessation and its associated factors in Vietnam
}

\author{
Le Thi Huong ${ }^{1 *}$, Hoang Van Minh ${ }^{1}$, Kim Bao Giang $^{1}$, Le Thi Thanh Xuan ${ }^{1}$, Vu Thi Thu Nga ${ }^{1}$, \\ Pham Quynh $\mathrm{Nga}^{2}$, Phan Thi Hai ${ }^{3}$, Jason Hsia ${ }^{4}$
}

${ }^{1}$ Institute for Preventive Medicine and Public Health, Hanoi Medical University, Hanoi, Vietnam;

*Corresponding Author: hathuhuong@yahoo.com

${ }^{2}$ World Health Organization Office in Vietnam, Hanoi, Vietnam

${ }^{3}$ Vietnam Steering Committee on Smoking and Health (VINACOSH), Hanoi, Vietnam

${ }^{4}$ Center for Disease Control and Prevention, Atlanta, USA

Received 4 July 2011; revised 25 July 2011; accepted 4 August 2011

\section{ABSTRACT}

Background: Even though the number of research on tobacco in Vietnam has recently increased rapidly, there remains a lack of reporting on the pattern of quit smoking and its correlates. It is necessary to have scientific evidence on this issue to help the policy makers and the Public Health sector to have appropriate intervention program to enhance the smoke cessation smoke program and improve the health status of the smokers. This study aims to: 1) Describe the pattern of smoking cessation in Vietnam 2010; 2) Examine the association between smoking cessation with some associated factors in Vietnam 2010. Methods: Data used in this paper were obtained from the Global Adult Tobacco Survey (GATS) conducted in Vietnam in 2010. A total of 11,142 households were selected for this survey using a two-phase sampling design analogous to a three-stage stratified cluster sampling. Both descriptive and analytical statistical analyses were carried out. Multivariate logistic regression modeling was performed to examine the association between the patterns of quit daily; quit smoke; attempt to quit and intend to quit among smokers with relevant factors. Results: The prevalence of the different type of cessation among male and female were $23.3 \%$ and $28.6 \%$ for quit daily; $19.1 \%$ and $1.0 \%$ for quit; $55.6 \%$ and $44.4 \%$ for attempt to quit and $29.6 \%$ and $19.3 \%$ for intend to quit respectively. The prevalence of quit daily and quit increased with age whereas attempt to quit decreased with age. Prevalence of cessation was highest among the group who were asked about smoking tobacco by health care providers. Some determinants of quit daily and quit include age, area, and knowledge of illnesses caused by smoking. There was no association between attempt and intend to quit with any socio-characteristic except for area. People in rural area were 2.1 and 1.7 times more likely attempt to quit and intend to quit than those in urban area, respectively. Conclusion: The GATS Vietnam 2010 showed that the prevalence of cessation in Vietnam was low. Quit daily and quit had association with age, area, knowledge about the top three consequences of smoke. People in rural area were 2.1 and 1.7 times more likely attempt to quit and intend to quit than those in urban area, respectively.

Keywords: Cessation; Socio-Demographic Factors; Global Adult Tobacco Use Survey; Vietnam

\section{INTRODUCTION}

Tobacco use was a major preventable cause of death in the world. Although many prevention attempts were made, the prevalence of tobacco smoking was still high in many countries. A study in the US indicated that four of the five hypothesized predictor variables had a significant relationship with intention to quit tobacco: gender, age, treatment modality, and smoking pattern. Age and gender demonstrated the strongest relationships to intention to quit smoking [1]. Results from another research suggested that parents' early smoking cessation has a long-term influence on their adult children's smoking cessation [2]. The majority of smokers made quit attempts without the benefit of proven behavioral or pharmacological treatments. Efforts were needed to increase use of smoking cessation treatments among all smokers, particularly combining treatments. Females, 
whites, older, more educated and wealthier smokers were more likely to adopt treatment in a quit attempt, as were more nicotine dependent smokers. Females were more likely than males to use behavioral treatments [3].

In Vietnam, current smoking prevalence estimates were $49.2 \%$ or higher among men, but less than $2 \%$ among women. Among male smokers in 2001-2002, $69.1 \%$ smoked cigarettes only, 23.2\% smoked water pipe tobacco only, and $7.7 \%$ reported using both products. $63 \%$ of households had at least one smoker. $71 \%$ of children under age 5 lived in households with at least one smoker [4]. In 2003, nearly 60\% school-attending youth reported being exposed to secondhand smoke at home [5]. The prevalence of cigarette smoking among students was $3.3 \%$ overall. The prevalence of smoking among male students (5.9\%) was higher than that among females (1.2\%) [5].

This study aims to:

- Describe the pattern of quit daily and quit smoking in Vietnam 2010.

- Examine the association between quit daily and quit with some associated factors in Vietnam 2010.

\section{METHODS}

Data used in this paper were obtained from the Global Adult Tobacco Survey (GATS) conducted in Vietnam in 2010. The GATS was a household survey that was launched in February 2007 as a new component of the ongoing Global Tobacco Surveillance System (GTSS) [6]. The GATS in Viet Nam was designed to be a nationally representative survey of all non-institutionalized men and women age 15 and older who considered Viet Nam to be their primary place of residence.

A total of 11,142 households were selected for this survey using a two-phase sampling design analogous to a three-stage stratified cluster sampling. The standard GATS questionnaire was designed to collect data on adult tobacco use and key tobacco control measures, including: 1) Tobacco use prevalence (smoking and smokeless tobacco products); 2) Second-hand smoke; 3) Cessation; 4) Knowledge, attitudes and perceptions; 5) Exposure to media; and 6) Economics. GATS questionnaire has been adapted to Vietnamese context and the final version of the questionnaire is approved by the GATS Questionnaire Review Committee for full survey.

Data collection was done by the General Statistics Office (GSO), under the co-supervision of the World Health Organization in Vietnam, Vinacosh and Hanoi Medical University. There were 26 data collection teams involved in GATS Vietnam 2010. Each team consisted of one team leader and 4 interviewers to ensure close supervision and collection of high quality data. They had computer skills and previous experience in conducting of GSO household-based surveys, especially GSO health- related surveys. In addition to the qualifications needed for interviewers, team leaders for the GATS were experienced in using computers and handheld (iPAQ) devices and had previous experience working with local authorities.

Handheld computers were used for capturing data. Each interviewer and team leader had one iPAQ. A real case file containing addresses and names of the households assigned to the interviewer was preloaded in the iPAQ prior to the field work. All the responses were entered in the iPAQ, with the help of a stylus for touching the key-pad on the screen. Data collection was conducted from 22 March 2010 to 13 May 2010 in all 63 provinces of Vietnam.

*Dependents variables using in this report includes:

Quit daily: The percentage of people who have been daily smokers in the past, but stopped for at least the past 12 months.

Numerator: Number of people who stopped smoking.

Denominator: Total number of daily smokers in the past.

Quit: The percentage of people who successfully quit smoking for the past 12 months.

Numerator: Number of people who stopped smoking.

Denominator: Total number of people who have been smokers in the past.

Attempt to quit: Percentage of adults who smoked tobacco during the past 12 months and tried to quit during the same period.

Numerator: Number of current tobacco smokers who tried to quit during the past 12 months and former tobacco smokers who have been abstinent for $<12$ months.

Denominator: Total number of current tobacco smokers and former tobacco smokers who have been abstinent for $<12$ months.

Intent to quit: Percentage of current tobacco smokers who are planning to quit or thinking about quitting.

Numerator: Number of current tobacco smokers who are planning or thinking about quitting smoking within the next month, 12 months, or eventually.

Denominator: Number of current tobacco smokers.

Intent to quit: Percentage of current tobacco smokers who are planning to quit or thinking about quitting.

Numerator: Number of current tobacco smokers who are planning or thinking about quitting smoking within the next month, 12 months, or eventually.

Denominator: Number of current tobacco smokers.

\section{RESULTS}

\subsection{General Characteristics}

Among the 11,142 sampled households, 10,383 were completely screened, giving a household response rate of 96.9\%. The household response rate was a little higher in rural areas compared to urban areas $(97.5 \%$ and $96.5 \%$, 
respectively). Overall, only $0.6 \%$ of the selected households refused to respond to the survey. Among 10,383 individuals selected from the completely screened households, 9925 were completely interviewed for a person-level response rate of $95.7 \%$. The person-level response rate was also a little higher in rural areas compared to that in urban areas $(96.3 \%$ and $95.0 \%$, respec- tively). In GATS Vietnam 2010, the total response rate was $92.7 \%$ (93.9\% in rural areas and $91.7 \%$ in urban sites).

Table 1 presented the sample size and population estimates by selected demographic characteristics. The 9925 completed interviews represented an estimated 64.3 million adults age 15 and over in Vietnam. By age group,

Table 1. Distribution of study subjects by selected socio-demographic characteristics—GATS Vietnam, 2010.

\begin{tabular}{|c|c|c|c|c|}
\hline & Characteristics & Sample size & Weighted number & Weighted \% \\
\hline \multicolumn{5}{|l|}{ Gender } \\
\hline & - Male & 4356 & $31,258,108$ & 48.6 \\
\hline & - Female & 5569 & $33,062,657$ & 51.4 \\
\hline \multicolumn{5}{|l|}{ Age } \\
\hline & - $15-24$ & 1656 & $16,637,021$ & 25.9 \\
\hline & - $25-34$ & 2053 & $12,661,740$ & 19.6 \\
\hline & - $35-44$ & 2198 & $14,281,840$ & 22.2 \\
\hline & - $45-54$ & 1867 & $9,657,483$ & 15.0 \\
\hline & - $55-64$ & 1019 & $5,407,631$ & 8.4 \\
\hline & - $>64$ & 1132 & $5,675,050$ & 8.8 \\
\hline Educatio & & - & & \\
\hline & - Primary & 2034 & $12,377,177$ & 26.0 \\
\hline & - Secondary & 3981 & $25,031,220$ & 52.5 \\
\hline & - High school & 1023 & $6,793,646$ & 14.3 \\
\hline & - College, university & 1227 & $3,447,042$ & 7.2 \\
\hline Occupati & tion & - & & \\
\hline & - Manager/Professional & 845,000 & $3,120,000$ & 6.6 \\
\hline & - Office Worker & 220,000 & 916,000 & 2.0 \\
\hline & - Service/Sales & $1,589,000$ & $8,991,000$ & 19.2 \\
\hline & - Farming & $3,069,000$ & $23,255,000$ & 49.6 \\
\hline & - Forestry/Fishing & 120,000 & 867,000 & 1.8 \\
\hline & - Construction/Mining & 317,000 & $2,442,000$ & 5.2 \\
\hline & - Production/Driving & 834,000 & $6,063,000$ & 12.9 \\
\hline & - Other & 248,000 & $1,272,000$ & 2.7 \\
\hline \multicolumn{5}{|c|}{ Ethnicity } \\
\hline & - Kinh & 8555 & $54,368,513$ & 84.5 \\
\hline & - Others & 1370 & $9,952,252$ & 15.5 \\
\hline \multicolumn{5}{|c|}{ Marital status } \\
\hline & - Single & 1882 & $16,846,557$ & 26.2 \\
\hline & - Married & 7078 & $43,452,453$ & 67.6 \\
\hline & - Separate & 67 & 218,162 & 0.3 \\
\hline & - Divorce & 152 & 556,605 & 0.9 \\
\hline & - Widow & 740 & $3,214,116$ & 5.0 \\
\hline Area & & - & & \\
\hline & - Urban & 4958 & $19,724,648$ & 30.7 \\
\hline & - Rural & 4967 & $44,596,117$ & 69.3 \\
\hline Total & & 9925 & $64,320,765$ & 100 \\
\hline
\end{tabular}


people age 25 - 44 made up the largest proportion (41.9\%) and those 65 and older accounted for the smallest share (8.8\%). The majority of the study population reported having lower secondary school education (52.5\%) and primary or less education (26.0\%). People with a college degree or above made up $7.2 \%$ of the study population. The main occupation of the study population was farmer (49.6\%), followed by service/ sales (19.2\%) and production/driving (12.9\%). Other occupations were manager/professional (6.6\%); construction/mining (5.2\%); office workers (2.0\%); forestry/fishing (1.8\%) and others (2.7\%). By ethnicity, $84.5 \%$ of the populations were Kinh People (the majority) and the remaining 15.5\% belonged to other ethnic minority groups. By marital status, $67.7 \%$ of the populations were married, $26.2 \%$ were still single and the remaining populations (6.2\%) were separate/divorce/widow. Two-thirds of people aged 15 and over in Vietnam were living in rural areas.

\subsection{Cessation and Demographic Characteristic}

Table 2 presented the pattern status of smoking cessation by different demographic characteristics. In general, most types of cessation (quit, attempt to quit and intend to quit) among male population were higher than that in the female population: $19.1 \%$ vs $1.0 \%$ for quit; $55.6 \%$ vs $44.4 \%$ for attempt to quit; and $29.6 \%$ vs $19.3 \%$ for intend to quit; however, male was lower than female for quit daily (23.3\% vs $28.6 \%$ ). Attempt to quit had the highest prevalence for both male and female group while quit had the lowest.

The prevalence of all types of cessation smoke was also higher in the rural population compared to the urban population (22.6\% vs $23.9 \%$ for quit daily; $9.2 \%$ vs $10.1 \%$ for quit; $51.9 \%$ vs $56.7 \%$ for attempt to quit and $25.7 \%$ vs $30.8 \%$ intend to quit). The percentage of the different types of quit was also higher among Kinh people than that in the other ethnic groups. Concerning marital status, the study found that there was no specific trend. The highest prevalence for quitting smoking was among the separated group (15.7\%), and lowest was among the single group, while quit daily had highest prevalence among the divorced group (41.2\%) and lowest among the single group (10\%). Attempt to quit and intend to quit was highest among the single and widow groups (36.8\%). Looking deep into different age groups, we can see that the prevalence of quit daily and quit increased with age from $2.8 \%$ and $6.4 \%$ for 15 - 24 year old up to $19 \%$ and $52.5 \%$ for $>64$ year old group while the attempt to quit decreased with age from $60.5 \%$ for group 15 - 24 to $47.7 \%$ for group $>64$ years old. There was no significant difference between groups in intend to quit. The highest prevalence was observed among the youngest group (15 - 24) and the lowest among oldest group (>64) with $34 \%$ and $22.5 \%$, respectively.

Table 2. The prevalence of cessation by demographic characteristics-GATS Vietnam, 2010.

\begin{tabular}{|c|c|c|c|c|}
\hline Characteristics & $\begin{array}{c}\text { Quit daily } \\
\% \text { (95\% CI) }\end{array}$ & $\begin{array}{c}\text { Quit } \\
\%(95 \% \text { CI) }\end{array}$ & $\begin{array}{l}\text { Attempt to quit } \\
\% \text { (95\% CI) }\end{array}$ & $\begin{array}{c}\text { Intend to quit } \\
\% \text { (95\% CI) }\end{array}$ \\
\hline \multicolumn{5}{|l|}{ Sex } \\
\hline Male & $23.3(21.7,24.9)$ & $19.1(18,20.3)$ & $55.6(53.6,57.7)$ & $29.6(27.7,31.5)$ \\
\hline Female & $28.6(20.2,36.9)$ & $1(0.8,1.3)$ & $44.4(34.2,54.5)$ & $19.3(11,27.7)$ \\
\hline$p$ value ${ }^{*}$ & $<0.001$ & $<0.001$ & $<0.001$ & $<0.001$ \\
\hline \multicolumn{5}{|l|}{ Residence } \\
\hline Urban & $22.6(20.4,24.9)$ & $9.2(8.4,10)$ & $51.9(49,54.8)$ & $25.7(23.1,28.3)$ \\
\hline Rural & $23.9(21.6,26.1)$ & $10.1(9.3,11)$ & 56.7 (53.9, 59.4) & $30.8(28.2,33.4)$ \\
\hline$p$ value ${ }^{*}$ & $<0.001$ & $<0.001$ & $<0.001$ & $<0.001$ \\
\hline \multicolumn{5}{|l|}{ Ethnic } \\
\hline Kinh & $25(23.3,26.8)$ & $10.3(9.6,10.9)$ & $57(54.8,59.2)$ & $30.5(28.4,32.6)$ \\
\hline Others & $15.6(12.1,19)$ & $7.5(6.1,8.9)$ & $47.6(42.8,52.3)$ & $23.8(19.7,28)$ \\
\hline$p$ value ${ }^{*}$ & $<0.001$ & $<0.001$ & $<0.001$ & $<0.001$ \\
\hline \multicolumn{5}{|l|}{ Marital status } \\
\hline Single & $10(6.4,13.7)$ & $4.1(3.2,5)$ & $61.8(56.4,67.2)$ & $36.8(31.2,42.4)$ \\
\hline Married & $25.2(23.4,27)$ & $12.3(11.5,13)$ & $54.5(52.3,56.7)$ & $28(25.9,30)$ \\
\hline Separate & $20.1(-10.1,50.3)$ & $15.7(6.8,24.7)$ & $25.8(-13.3,64.9)$ & $20.6(-15.5,56.8)$ \\
\hline Divorce & $12.5(1.3,23.7)$ & $3.9(0.8,7.1)$ & $51.3(33,69.6)$ & $18.2(3.6,32.9)$ \\
\hline Widow & $10(6.4,13.7)$ & $4.1(3.2,5)$ & $61.8(56.4,67.2)$ & $36.8(31.2,42.4)$ \\
\hline$p$ value ${ }^{*}$ & $<0.001$ & $<0.001$ & $<0.001$ & $<0.001$ \\
\hline
\end{tabular}


Figure 1 showed that the trend of all kind of cessation increased by education level. It meant that higher education level had higher concern in cessation. The primary education or less had lowest percentage of quit daily and quit $(20.2 \%$ and $8.2 \%)$ while this number in the college and above group was (31.5\% and $15.9 \%)$. There was a similarity in intend to quit in upper secondary, college and above groups (around $40 \%$ ). The attempt to quit was similar among the last three groups (56.2\%; 59\% and $56.2 \%$ for lower secondary, upper secondary and college respectively), while this number among the primary education group was $46.1 \%$.

This study indicated that the manager and officer workers had highest prevalence of all kind of cessation and the forestry/fishing had lowest prevalence of all kind of cessation. Quit daily was highest prevalence in office worker group (44.7\%), followed by the group of managers/professional (32\%); then service/sales and farming (27.3\% and $21.3 \%)$. Forestry/fishing was a group with lowest prevalence of quit daily (6\% only). Quit was highest among manager/professional (17.5\%), followed by officer worker (13.6\%) and construction and mining
(12.3\%) then farmer (10.4\%) and services/sales (10.1\%); the lowest prevalence belonged to the group of production/driving (8.8\%) and forestry/fishing (7.0\%). Attempt to quit was highest among group of officer worker (66.7\%); followed by construction/mining group and manager group (60.5\% and $60.3 \%$ respectively). The lowest prevalence of attempt to quit belonged to forestry/fishing as well. The same trend also observed for intend to quit. The highest prevalence was officer worker and manager groups $(41.4 \%$ and $42.2 \%$ respectively) while in the forestry group this prevalence was $17.8 \%$.

\subsection{Cessation and Health Services}

Figure 2 shows that the prevalence of cessation was highest among the group of asked about smoking tobacco by health care providers (34.9\%) followed by the group with care provider's advice to quit tobacco smoking (29.7\%) and lowest prevalence of cessation was belong to the group visited health-care providers. This means that the interaction between health care providers and smokers is affecting the cessation status.

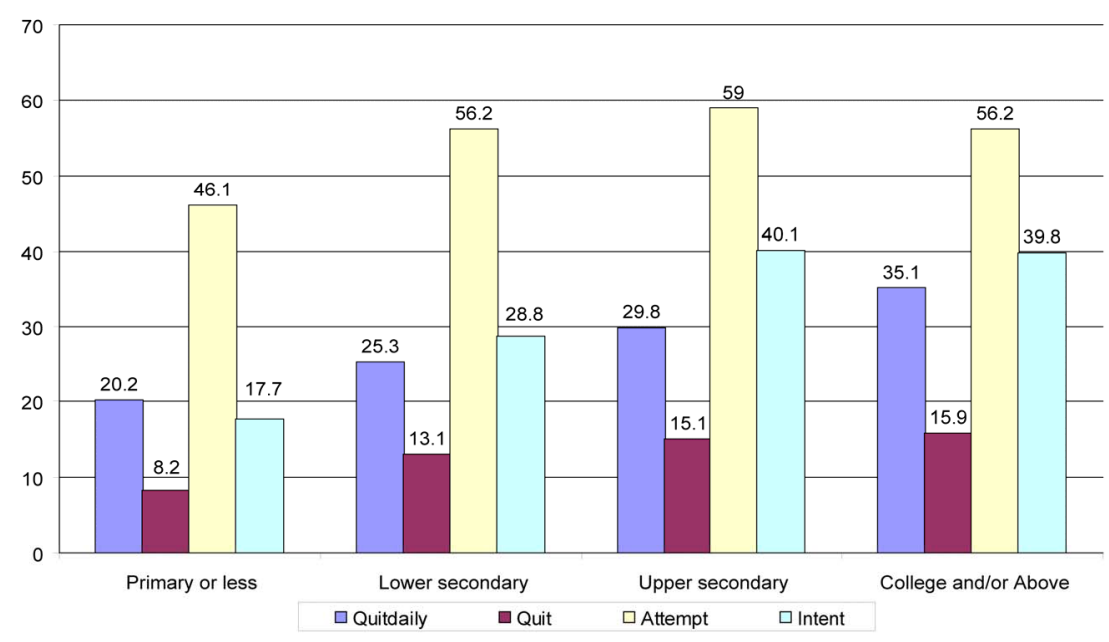

Figure 1. Prevalence cessation by education (>25 years old)_-Vietnam GATS, 2010.

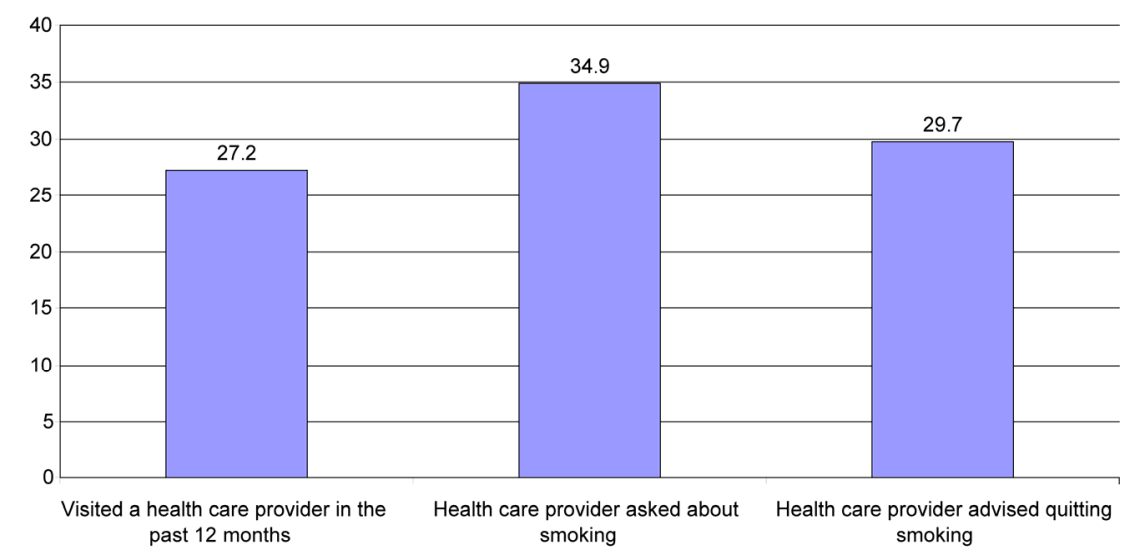

Figure 2. Prevalence of cessation by health services-Vietnam GATS, 2010. 


\subsection{Determinant Factors of Cessation}

Table 3 showed that gender, educational level, ethnicity, marital status, region had no association with the quit daily status. However, the quit daily trend increased with age. People at age group 25 - 35; 35 - 45; 45 - 55 and $>55$ had quit daily prevalence at 2.3, 3.2, 3.5 and 5.7 times, respectively, higher than age group of $15-25$. People living in rural had 1.8 times higher in quit daily prevalence than those urban. People who knew about the

Table 3. Logistic regression analysis of the association between cessation with selected socio-demographic factors-Vietnam GATS, 2010.

\begin{tabular}{|c|c|c|c|c|c|}
\hline & & \multicolumn{4}{|c|}{ OR (95\% CI) } \\
\hline & & Quit daily & Quit & attempts to quit & Intend to quit \\
\hline \multicolumn{6}{|l|}{ Gender } \\
\hline & Male & 1 & 1 & 1 & 1 \\
\hline & Female & $1.1[0.6-2]$ & $0.034[0.025-0.048]$ & $1.2[0.3-4.6]$ & $0.9[0.1-5.4]$ \\
\hline \multicolumn{6}{|c|}{ Age group } \\
\hline & $15-25$ & 1 & 1 & 1 & 1 \\
\hline & $25-34$ & $2.3[1-5.5]$ & $2.6[1.5-4.3]$ & $1[0.3-3.7]$ & $0.8[0.2-2.8]$ \\
\hline & $35-44$ & $3.2[1.4-7.4]$ & $4.4[2.7-7.4]$ & $1.1[0.3-3.9]$ & $0.9[0.3-3.2]$ \\
\hline & $45-55$ & $3.5[1.5-8.2]$ & $5.3[3.2-8.9]$ & $1[0.3-3.8]$ & $0.9[0.2-3.3]$ \\
\hline • & $>55$ & $5.7[2.4-13.7]$ & $8.4[5.1-13.8]$ & $0.7[0.2-3]$ & $0.4[0.1-1.7]$ \\
\hline
\end{tabular}

Education

- Primary or less

- Lower secondary

- Upper secondary

- College and/or above

Ethnic

- Kinh

- Others

Marital

- $\quad$ Maried

- Singer

- Separate/divorce

- Widow

Area

- Urban

- Rural

Believes that tobacco smoking causes strokes and heart attacks and lung caner

- No

- Yes

Believes that secondhand smoke causes serious

illness in non-smokers
1

- Yes

\section{1}

$0.8[0.6-1.3]$

1

$0.7[0.4-1.2]$

0.5 [0.2 - 1.3]

$1.2[0.7-2.2]$

1

$1.8[1.4-2.4]$

1

$1.7[1.3-2.1]$

1

$1.4[1.2-1.8]$

1

$1.2[0.9-1.7]$
1

$1.5[0.7-3.2] \quad 3.4[1.3-8.7]$ 
top three consequences of smoking had 1.7 times higher prevalence of quit daily than people who did not know. Logistic regression also showed that educational level, job, ethnicity, marital status, region, have no association with the quit group. Gender, age, area, knowing about the top three consequences of smoking and knowing about danger of the SHS had an association with quit smoking. Quit smoking increased with age group. People at age group 25 - 35; 35 - 45; 45 - 55 and >55 had quit prevalence at 2.6, 4.4, 5.3 and 8.4 times, respectively, higher than age group of 15 - 25. Female group had prevalence of smoking equal to 0.034 of that in male group. Rural area had 1.4 times higher in quit smoke than that in urban. People who knew about the top three consequences of smoking had 1.4 times higher in prevalence of quit smoke than people who did not know and people who knew about the dangers of the SHS had 1.2 times higher in quit smoke than people who did not know. Except for area characteristic, there was no association between attempt to quit with any characteristic. Rural people attempted to quit 2.1 times more than urban people. The same results observed for intend to quit, except for the association between areas with intend to quit, there was no related socio-demographic factors found. Rural people have 1.7 times higher in intend to quit than urban people.

\section{DISCUSSION}

It was interesting that except for quit daily, the prevalence of quit, attempt to quit and intend to quit among male population was higher than that in the female population study: $19.1 \%$ vs. $1.0 \%$ for quit; $55.6 \%$ vs. $44.4 \%$ for attempt to quit and $29.6 \%$ vs. $19.3 \%$ for intend to quit. Attempt to quit was highest prevalence for both male and female group (55.6\% and 44\%) while the lowest one was quit $(19.1 \%$ and $1 \%$ for male and female respectively). When we looked at the logistic regression, there was only one association between gender and quit, the prevalence of quit among female was only equal to 0.034 of that in male group. Hackshaw $\mathrm{L}$ et al mentioned in their study that "there were no significant differences in these quit attempts with regard to gender, socio grade or cigarette consumption" [7]. However, another study among in-school adolescents in Ukraine indicated that while male adolescents who received support on how to stop smoking from a family member were $7 \%$ less likely, female adolescents were $60 \%$ more likely to stop smoking. Furthermore, while male adolescents who received a lecture on the harmful effects of smoking were $10 \%$ less likely, female adolescents were $9 \%$ more likely to stop smoking [8]. In Viet Nam the situation may be different, for the Vietnamese culture, smoking among women was not popular and most smoking women had special circumstances, therefore it might be difficult for women than men to have cessation decision. On the other hand women might smoke fewer cigarettes than men, so they might think that the consequences were not as serious. Another study with 224 randomly interviewed patients, $64.3 \%$ were women with the mean age of 44.9 . Rate of smokers was $17 \%$ (52.63\% female). There were younger female smokers than male, but females smoked fewer cigarettes/day. Only half of the smokers were motivated to stop smoking [9].

The prevalence of all kind of smoking cessation was also lower in the urban population compared to the rural population (22.6\% vs. $23.9 \%$ for quit daily; $9.2 \%$ vs. $10.1 \%$ for quit; $51.9 \%$ vs. $56.7 \%$ for attempt to quit and $25.7 \%$ vs. $30.8 \%$ intend to quit). In the logistic model, the people from rural were at $1.8 ; 1.4 ; 2.8$ and 1.7 times higher in quit daily, quit, attempt to quit and intend to quit than urban people. Our finding was different with the findings from Thailand GATS report. In Thailand, current smokers living in urban areas have higher rate of cessation than rural area [10].

Data from this study indicated that the cessation prevalence increased with age. In the logistic regression, people at age group 25 - 35; 35 - 45; 45 - 55 and $>55$ had quit prevalence at 2.6, 4.4, 5.3 and 8.4 times respectively higher than age group of 15 - 25. This finding was also in line with the finding from the study in England in 2010 that there was a significant linear trend of quit attempt with increasing age [7]. A study in Japan also showed similar results that those smoking more cigarettes and who started smoking at a young age were less likely to stop smoking. Older age, physical activity, and participation in health checkups were associated with smoking cessation [11]. However, Solberg LI et al. indicated in their study that higher rates of smoking among young adults do not reflect less interest in quitting, fewer quit attempts, or less success in quitting compared with older adults [12].

In this study, higher education has higher prevalence of cessation especially quit daily and quit. This was also the same findings as the GATS study reported in Thailand, which was mentioned in this report that current smokers with a university level of education had a higher rate of planning to quit within the next month (12.2\%) compared to current smokers with a primary level of education (6.2\%) [10].

People who knew about the top three consequences of smoking had 1.4 times higher in prevalence of quit daily than people who did not know and those who knew about the dangers of the SHS had 1.2 times higher in quit daily than people who did not know. This meant that the knowledge of people regarding to the harmful effects of smoking affected their cessation status. Therefore it was necessary to pay more attention to increase the awareness of the community on the cause and consequences of 
smoking to encourage cessation. These findings also agreed with findings from other studies in Thailand and Japan $[10,11]$.

In our study, the prevalence of cessation was highest among the group who were being asked about smoking tobacco by HCP (34.9\%) followed by the group with HCP's advice to quit tobacco smoking (29.7\%) and lowest prevalence of cessation belonged to the group visited health-care providers. It was difficult for most tobacco users to quit on their own and they would benefit from help and support to overcome their dependence. The WHO FCTC recommended in Article 14, "demand reduction measures concerning tobacco dependence and cessation" that Parties implement best practices to promote cessation of tobacco use and implement the treatment of nicotine addiction [13]. MPOWER also emphasizeed "offering help to quit tobacco use" and recommended three types of tobacco dependence treatment: 1) tobacco cessation advice incorporated into primary healthcare services; 2) easily accessible and free quit lines; and 3) access to low-cost pharmacological therapy [14]. Therefore it was necessary to have effective cessation services in cessation clinics and implementation of quit lines.

\subsection{In Conclusions}

The GATS Vietnam 2010 showed that the prevalence of cessation in Vietnam was low. Quit daily and quit had association with age, area, knowledge about the top three consequences of smoking. Rural people are more likely attempt to quit and intend to quit 2.1 and 1.7 times than urban people.

\subsection{Copyright License Statement}

This study was financial supported by WHO and technical supported by CDC. This paper was partly funded by the project "Public health preparedness and response to critical global health issues in Vietnam and Sweden" which is being implemented by Epidemiology and Global Health, Umeå University, Sweden \& Hanoi Medical University, Vietnam. They allowed this paper be published without any competing interest.

\section{REFERENCES}

[1] Moore, D., Langlois, M., Gerber, B., Gaddis, R., Hallam,
J. and Arnold, R. (2007) Intention to quit tobacco use among clients in substance use disorder treatment settings. Substance Use \& Misuse, 42, 871-879. doi:10.1080/10826080701202528

[2] Bricker, J., Otten, R. and Liu, J. (2009) Parents who quit smoking and their adult children's smoking cessation: A 20-year follow-up study. Addiction, 104, 1036-1042.

[3] Shiffman, S., Brockwell, S., Pillitteri, J. and Gitchell, J. (2008) Individual differences in adoption of treatment for smoking cessation: Demographic and smoking history characteristics. Drug and Alcohol Dependence, 93, 121131. doi:10.1016/j.drugalcdep.2007.09.005

[4] Ministry of Health (2003) Vietnam national health survey (VNHS). Ministry of Health, Hanoi.

[5] Centers for Disease Control and Prevention (2003) Global youth tobacco survey (GYTS) 2003. Centers for Disease Control and Prevention, Atlanta.

[6] Centers for Disease Control and Prevention (2007) Global youth tobacco survey (GYTS) 2007. Centers for Disease Control and Prevention, Atlanta.

[7] Hackshaw, L.M.A., West, R. and Bauld, L. (2010) Quit attempts in response to smoke-free legislation in England. Tobacco Control, 19, 160-164. doi:10.1136/tc.2009.032656

[8] Hazemba, A.S.S., Muula, A.S. and Rudatsikira, E. (2010) Gender specific factors associated with having stopped smoking among in-school adolescents in Ukraine: Results from the Global Youth Tobacco Survey 2005. BMC Research Notes, 16, 76. doi:10.1186/1756-0500-3-76

[9] Rosendo, I., Fonseca, G., Guedes, A. and Martins, V.A. (2009) Characterisation of smokers and factors influencing motivation to stop smoking. Revista Portuguesa de Pneumologia, 15, 783-802.

[10] WHO (2009) Global adult tobaco survey (GATS): Thailand country report.

http://wwwsearowhoint/LinkFiles/Regional_Tobacco_Sur veillance_System_GATS_Thailand_2009pdf

[11] Honjo, K.I.H., Inoue, M., Tsugane, S. and JPHC Study Group (2010) Smoking cessation: Predictive factors among middle-aged Japanese. Nicotine \& Tobacco Research, 12, 1050-1054. doi:10.1093/ntr/ntq143

[12] Solberg, L.I.B.R., McCarty, M., Asche, S.E. and Thoele, M.J. (2007) Young adult smokers: Are they different. American Journal of Managed Care, 13, 626-632.

[13] World Health Organization (2003) WHO framework convention on tobacco control. World Health Organization, Geneva.

[14] World Health Organization (2008) WHO report on the global tobacco epidemic. World Health Organization, Geneva. 